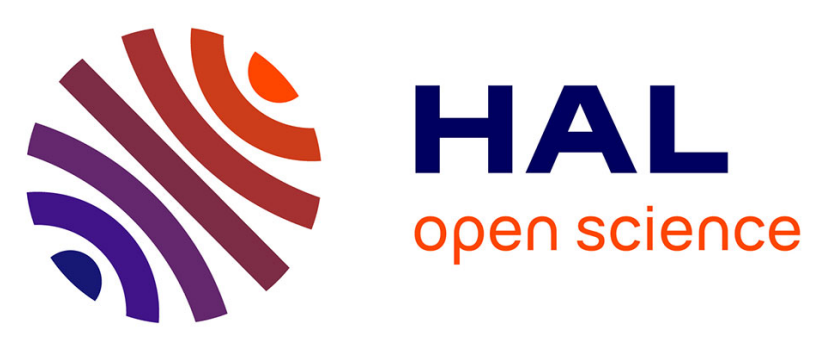

\title{
An RML algorithm for retrieval of sinusoids with cascaded notch filters
}

Nacer K M'Sirdi, Ioan Doré Landau

\section{To cite this version:}

Nacer K M'Sirdi, Ioan Doré Landau. An RML algorithm for retrieval of sinusoids with cascaded notch filters. ICASSP-88., International Conference on Acoustics, Speech, and Signal Processing, New York, NY, USA, 1988, pp. 2484-2487 vol.4., Sep 1988, Grenoble, France. hal-02482009

\section{HAL Id: hal-02482009 \\ https://hal-amu.archives-ouvertes.fr/hal-02482009}

Submitted on 17 Feb 2020

HAL is a multi-disciplinary open access archive for the deposit and dissemination of scientific research documents, whether they are published or not. The documents may come from teaching and research institutions in France or abroad, or from public or private research centers.
L'archive ouverte pluridisciplinaire HAL, est destinée au dépôt et à la diffusion de documents scientifiques de niveau recherche, publiés ou non, émanant des établissements d'enseignement et de recherche français ou étrangers, des laboratoires publics ou privés. 


\title{
AN RML ALGORITHM FOR RETRIEVAL OF SINUSOIDS WITH CASCADED NOTCH FILTERS
}

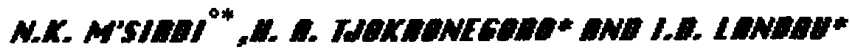 \\ - LaBORATORE D'aUTOMATIQUE ET ROBOTIQUE UniY. P. M. CURE (PARES 6) \\ * LABORATOIRE D'AUTOMATYUE DE GRENOBLE (I.N.P.G.) \\ 4 GRECO BYSTENES ADAPTATIFS (C.N.R.S] \\ [E.N.S.I.E.G.) B.P. 40 - 39402 8T MARTR D'HERES FRALCE
}

\section{ABSTRACT:}

To provide the frequencies estimation, Adaptive Notch Filters [1-4] can be used by implementation in a cascad blocks of second order cells. Some strategies to track time varying parameters can be used [6]. Then, in a second step the estimated frequencies are used to provide the estimation of amplitudes and phases in a recursive manner. The proposed recursive algorithm consistsin two steps. The first step involves a Maximum Likelihood algorithm to adapt the cascaded filters parameters [1][4], which will provide the frequencies estimates. The second step uses the last estimates, and then the estimations of amplitudes and phases are given by a Recursive Least Squares algorithm[6]. The proposed algorithm is asymptotically consistent and robust faced with the neglected dynamics. In case of time varying signals, its tracking capabilities insure the goodness of the estimations. The accuracy of estimation is better with the RML than with the ELS method for which an opper limit of the debiasing parameter is crucial in order to have a convergencewithoutlocalinstabilities

\section{I-INTRODUCTION}

Our main interest in this contribution, is the retrieval of sinusoidal signals in noise. AR and ARMA modelling techniques have powerfull potentialities and wellsuited for signal spectrum analysis. However, when the noise level is high and / or when the signal model has poles near or on the unit circle, serious problems arise[1]. In the narrowband case, successfull applications have been realized with AR modelling [2-3]. The high order autoregressve FR filter is shown to be asymptotically equivalent to an Adaptive Notch Filter (ANF). The noise effect is reduced by increasing the order of of the AR filter at the expense of computational cost. Thus the a priori knowlege of a narrowband leads to the Notch Filter Structure (NFS) with constrained poles and zeros. This has recently been proposed in extensive simulations [4-7]. A theoretical analysis have been presented in [5][7] and the applicable estimation methods are reviewed in [8].
These last structures introduce in ParameterAdaptation Algorithms (PAA) some nolinearity which may give some problems in the transient period of the estimation. The cascade implementation of second order have been claimed possible. Fast Least Squares (FLS) estimation algorithm case have been considered and studied in simulations [9]. The main interest of the ANF Cascade form is to simplify computation of the estimated frequencies and in case of independly time varyingfrequencies, a second order filter appears faster to track variation than a higher order one.

In section Il of this paper we develop an implementation of cascaded ANF. The Prediction Error Method (PEM) is applied leading to a Recursive Maximum Likelihood (RML) algorithm. This PAA allows the computation of the estimated frequencies. In a second step, using these estimates, a Recursive Least Squares (RLS) algorithm is employed for the estimation of amplitudes and phases. The analysis and performance evaluation of these algorithms is presented in section III.

\section{II-Recursive estimation algorithm and} filter structure.

\subsection{Frequeacy estimation whit cascaded ANF}

The recursive estimation procedure consists of two stages. The first one involves an $\mathrm{ANF}$ in cascade form for the estimation of the frequencies. The other one uses an adaptive algorithm to provide the amplitudes and phases estimations. The involved signals may be modeled as follows where $v_{k}$ is the noise disturbance:

$$
y_{k}=\sum_{k=1}^{p} c_{i} \sin \left(\omega_{i} \cdot k+\beta_{j}\right)+v_{k}
$$

Adaptive Notch Filters are very well suited for estimation of the sinusoidal component frequencies. Let us consider the notch filter transfert functionincascade form

$$
\mathrm{H}\left(\mathrm{z}^{-1}\right)=\prod_{i=1}^{p} \mathrm{H}_{\mathrm{i}}\left(\mathrm{z}^{-1}\right)=\prod_{i=1}^{p}\left[\left(1+\mathrm{a}_{\mathrm{i}} \mathrm{z}^{-1}+\mathrm{z}^{-2}\right) /\left(1+\mathrm{a}_{j} \mathrm{z}^{-1}+\mathrm{r}^{2} \mathrm{z}^{-2}\right)\right] \text { (2) }
$$

With no loss of generality we assume identical bandwidth 
for the notches. If the frequencies are all independant we can write for all $\mathrm{j}=1 \ldots \mathrm{p}$

$$
\tilde{y}_{k}^{i}=\prod_{\substack{i=1 \\ i \neq j}}^{p} H_{i}\left(q^{-1}\right) \cdot y_{k}
$$

and the prediction error is: $\varepsilon_{k}=H_{j}\left(q^{-1}\right) \tilde{y}_{k}^{j}$

The last equation presents a second order notch filter. Thus independence between frequencies yields independence between parameters $a_{i}$ of each second order cell. Then each cellcan be adapted independently of the others afterprefiltering the signal by the others. If we suppose that the filters $\mathrm{H}_{\mathrm{i}}\left(\mathrm{z}^{-1}\right)$ with $\mathrm{j}$ and $\mathrm{i}=1 \ldots \mathrm{p}$ have converged then the filter $\mathrm{H}_{\mathrm{j}}\left(\mathrm{z}^{-1}\right)$ will remove the remaining component. Thus there exits a unique global minimum for the quadratic criterion. Following the Prediction Error technique for the estimationalgorithm, weobtain thegradient:

$$
\psi_{k-1}^{j}=-\frac{d \varepsilon_{k}}{d a_{j}}=\frac{(1-r)\left(1-r q^{-2}\right)}{\left(1+r \cdot a_{j} q^{-1}+r^{2} q^{-2}\right)^{2}} \tilde{y}_{k-1}^{j}(5)
$$

The RML algorithm may be summarized by the following equations:

$$
\begin{aligned}
& \hat{a}_{j}(k)=\hat{a}_{j}(k-1)+F_{k-1} \psi_{k-1}^{j} \varepsilon_{k}^{j} \\
& F_{k}^{\prime}=F_{k-1}^{\prime} /\left(\lambda+F_{k-1} \psi_{k-1}^{2}\right)
\end{aligned}
$$

An approximation must be done to reduce computational complexity. It yields an Approximate RML algorithm. The computational complexity is essentally du to the computation of the gradient. We can then use the formula:

$$
\psi_{k-1}^{j}=\frac{f_{j}(k-1)-r \cdot \varepsilon_{k-1}}{1+r a_{j} q^{-1}+r^{2} q^{-2}}
$$

Withf $(\mathbf{j}-1)$ computed as follows:

$$
\begin{aligned}
& y_{k}^{j}=\prod_{l=j+1}^{p} H_{l}\left(q^{-1}\right) y_{k} ; j=p-1, \ldots, 1 \\
& f_{j}(k)=y_{k}^{j}-y_{k}^{j-1}
\end{aligned}
$$

\subsection{Amplitude and phase estimation with WRLS:}

In this second step we assume the frequencies known and use a Weighted Recursive Least Squares algorithm to estimate the amplitudes and phases as in [10]. The frequency estimates are provided by the first step.

$$
\begin{aligned}
& \text { Model (1) of the signal may be written: } \\
& y_{k}=\sum_{l=1}^{p}\left(g_{l} \cos \omega_{l} \cdot k+h_{\ell} \sin \omega_{l} \cdot k\right)+v_{k}
\end{aligned}
$$

where the amplitudes $C_{\ell}$ and phases $\beta_{\ell}(\ell=1 \ldots p)$ are given by:

$$
c_{l}=\left(g_{l}^{2}+h_{l}^{2}\right)^{1 / 2} \text { and } \operatorname{tg} \beta=\frac{g_{l}}{h_{e}}
$$

We can define the parameter vector and the observation vector as:

$$
\text { and } \begin{aligned}
\phi_{k} & =\left[g_{1}, \ldots, g_{p}, h_{1}, \ldots, h_{p}\right]^{\top} \\
\phi_{k} & =\left[\cos \omega_{1} k, ., \cos \omega_{p}, \sin \omega_{1} k, ., \sin \omega_{p} k\right]^{\top}
\end{aligned}
$$

The parameter vector is estimated with the WRLS algorithm:

$$
\begin{aligned}
& \varepsilon_{k}^{0}=y_{k}-\hat{\theta}_{k-1}^{\top} \phi_{k} \\
& F_{k}^{\prime}=\frac{1}{\lambda}\left[F_{k-1}-\frac{F_{k-1} \phi_{k} \phi_{k}^{T} F_{k-1}}{\lambda+\phi_{k}^{\top} F_{k-1} \phi_{k}}\right] \\
& \hat{\theta}_{k}=\hat{\theta}_{k-1}+F_{k} \phi_{k}^{\infty} \varepsilon_{k}^{o}
\end{aligned}
$$

In equation (12) the filtered version of the signal produced by the first stage, can be used.

\section{III- Analysis and Performance Evaluation}

\subsection{Convergence of the ANF}

\section{a) Second order ANF (RML)}

Let us first consider the case of a single cell $\mathrm{H}_{\mathrm{j}}(\mathrm{z})$ (see (4)) driven by the signal defined in (3) and assume the filters $\mathrm{H}_{\mathrm{i}}(\mathrm{z})$ $i=1 . j-1, j+1, . . n$ have converged to their optimal value. Thus $\tilde{y}_{k} j$ is composed by a single frequency $\omega_{\mathrm{j}}$ plus additive independant noise. The bandwidth are large enough to remove(or attenuate) the other frequencies.

Taking for the signal an AR model with poles on the unit circle and applying the RML algorithm, the a posteriori error can be written:

$$
\begin{aligned}
& \varepsilon_{k}=\frac{\hat{A}\left(r q^{-1}\right)}{A\left(r q^{-1}\right)}\left(\hat{a}_{j}-a_{j}\right) \psi_{k-1}^{j}+\frac{v_{k}}{A\left(r q^{-1}\right)} \\
& \text { with } \tilde{y}_{k}^{j}=-a_{j} \tilde{y}_{k-1}^{j}-\tilde{y}_{k-2}^{j}+v_{k} \\
& \text { and } \quad \begin{array}{l}
A\left(r q^{-1}\right)=1+r \cdot a_{j} q^{-1}+r^{2} q^{-2} \\
\hat{A}\left(r q^{-1}\right)=1+r \cdot \hat{a}_{j} q^{-1}+r^{2} q^{-2}
\end{array}
\end{aligned}
$$

It is well known that the RML algorithm needs astability monitoring in general case. For the ANF this procedure can be removed in virtu of the following Lemma.

Lemma 1: Let $r$ by exponentially time varying, from 0 to one, according to: $r_{k}=r_{d} \cdot r_{k-1}+\left(1-r_{0}\right) \cdot r_{f}$,

Then there exit an $r_{d}, r_{0}$ and $r_{f}$ such that $\hat{A}\left(r_{k} \cdot q^{-1}\right)$ is infinitely often stable.

proof : we can take $r_{\mathrm{f}}<$ inverse of the maximum modulus of the unstable poles of $A\left(q^{-1}\right)$.

Now applying the theoretical bakground in [11] and in particular Theorem 4 of [11] leads to the following: 
Theorem 1: Under the assumptions

A1: is infinitely often stable

A2: for some $\hat{a}_{j}$ fixed $\Psi_{k}^{j}, \mathcal{E}_{k}, w_{k}=\frac{v_{k}}{A_{r-t^{-1}}}$ are stationary

A3: $\omega_{k}$ is independent of $\psi_{k-1}^{j} \quad A$

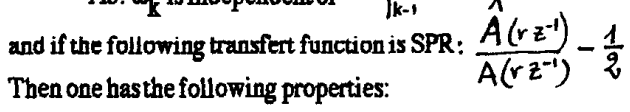

$$
\begin{aligned}
& \text { Convergence Domain. } \\
& \text { P1: } \lim \left(\hat{\varepsilon}_{\mathrm{k}}-()_{\mathrm{k}}\right)=0 \mathrm{wp} 1 \\
& \text { P2: } \lim \left(\hat{a}_{\mathrm{j}}\right) \in \mathrm{D}_{\mathrm{c}} \mathrm{wp} 1
\end{aligned}
$$$$
\text { with } D_{c}=\left\{a /\left(a-\hat{a}_{j}\right) \psi_{k-1}^{d}=0\right\}
$$

where $D_{c}$ is theconvergence domain

Leman 2: $\forall \hat{a}_{j, j}$ aj there exists an $r$ such that $\frac{\hat{A}}{A}-\frac{1}{8}$ is SPR.

The last condition on $r$ is less restrictive than the one for the stability. Lemmas 1 and 2 allow usto assert the global stability of the ANF if $r$ is appropriately chosen such that $\hat{A}\left(r \cdot q^{-1}\right)$ is always stable duringestimation.

\section{b) ELS parameter adaptation algorithm:}

If the ELSestimation method is applied (13) becomes [5]:

$$
\varepsilon_{k}=\frac{1}{A\left(r q^{-1}\right)}\left(\hat{a}_{j}-a_{j}\right) \psi_{k-1}^{j}+w_{k}
$$

The SPR condition is more restrictive than the stability one on $r$ and Theorem 1 cannot be applied in all cases. There exits an upper limit $l$ for the debiasing parameter in order to have the SPR condition. This limit $\mathcal{P}$ depend of the signal frequency and limits the accuracy of theestimates.

Thus the local stability cannot be etablished although the global stability canbeensured.

\section{c) Uniqueness of the RML estimates:}

In theorem 1 we have asymptotically wp1 (P1):

$$
\varepsilon_{k}=\frac{1}{A\left(r q^{-1}\right)} v_{k}
$$

Equation (4) and (14) yield:

$$
\varepsilon_{k}=\frac{\hat{A}\left(q^{-1}\right)}{\hat{A}\left(q^{-1}\right)} \tilde{y}_{k}^{j}=\frac{\hat{A}\left(q^{-1}\right)}{\hat{A}\left(r q^{-1}\right)} \cdot \frac{v_{k}}{A\left(q^{-1}\right)}
$$

It follows from (17) and (18):

$$
\frac{\hat{A}\left(q^{-1}\right)}{\hat{A}\left(r q^{-1}\right)}=\frac{A\left(q^{-1}\right)}{A\left(r q^{-1}\right)}
$$

and then $\hat{a}_{1}=a_{1}$ with probability one (wpl) if $r \neq 1$.

Theoren 2: If the order is correct the RML estimates for ANF are unique.

The main resultsconserning ANF are :

i) the global convergence can be ensured with an appropriate choice of the debiasing parameter without anystability monitoring

ii) The RML estimates are unique.

For $p$ cells in cascade adapted along (3_6), each cell will converge near to a local minimum [5]. The filtering (3) will make these minima distinctremowing, for each cell, the other frequencies.

\subsection{Amplitude and phase estimates:}

Exponential convergence of the amplitude and phase

estimates is garanteed by the following theorem which uses the conceptof persistentexcitation [12].

Theorem 3: The RLS estimation scheme (12) with exponential forgetting factorisexponentiallystable.

The proof of this theorem can be conducted in the same lines as in [12]. The regressors are composed by $p$ sinusoids having different frequencies. Then the persistent excitation condition is satisfyed:

$$
0<\alpha I \leqslant \sum_{k=j}^{j+s} \phi_{k} \phi_{k}^{\top} \leqslant \beta I<\infty
$$

Equation (20) yields by lemma 1 of [12]

$$
0<\frac{\alpha\left(\lambda^{-1}-1\right)}{\lambda^{-(s+1)}-1} I \leqslant F_{k-1}^{-1} \leqslant \frac{\beta}{1-\lambda^{s+1}}+0\left(\lambda^{k}\right)(21)
$$

The adaptation $\sqrt{\text { is }}$ bounded and will never be zero (tracking capabilities). Finally reasoning asin [12] we obtain.

$$
\left\|\hat{q}_{k}\right\|^{2} \leqslant \frac{\lambda^{-(s+1)}-1}{\alpha\left(\lambda^{-1}-1\right)} \lambda^{k} \cdot \lambda_{\max }\left(F_{0}^{-1}\right)\left\|q_{0}\right\|^{2}(22)
$$

\section{IV-Conclusion}

The main results demonstrate the good performances observed in simulation for the ANF. The exponential convergence of the WRLS estimates of amplitudes and phases is proved by use of the theoretical backgroung on persistentexcitation[12].

These results are very important for the time varying systems or in case of neglected dynamics due to the resulting robustness of the algorithms. The implementation form studied here is computationally attractive and robust also when frequency, amplitude or phase istime varying .

\section{Atrowlegement}

The authors want to tank $O$. Macchi for the hel pfull discussions. 


\section{R E F E R EN C E S}

[1] I. D. LANDAU, N. K. M'SRDI and M. M'SAAD. Techniques de modelisation recursive pour l'analyse spectrale parametrique adaptative. Traitement do signal, Vol 3, $\mathrm{N}^{\circ} 4-5,1986$.

[2] B. WIDROW et al.(1975). Adaptive noise cancelling: Principles and applications. Proc. IEEE, V63, Dec. 1976

[3] K. GLOVER. (1977). Adaptive Noise Cancelling applied to sinusoidal interferences. IEEE ASSP, V25, N6

[4] A. NEHORAI (1985). A minimal parameter adaptive notch filter with constrainedpoles and zeros. IEEE Trans. ASSP, V33, pp 983-996, August 1985.

[5] N.K. M'SIRDI et I.D. LANDAU (1985). Analyse spectrale adaptative de signaux a bandes étroites. Rapport interne 86_70. L.A.G. (E.N.S.I.E.G) St Martin d'Héres 38402. FRANCE

[6] J. M. T. ROMANO and M. BELLANGER (1987). Fast least squares adaptive notch filtering. 8th conference ECCTD, 1 - 4 September 1987, PARIS - FRANCE.

[7] D.V. BASKAR RAO and S.Y. KUNG (1984). Adaptive notch filtering for retrieval of sinusoids in noise, IEEE Trans. ASSP, Vol. 32, $\mathrm{N}^{0} 4$, August 1984.

[8] N.K. M'SIRDI et I.D. LANDAU (1987). Adaptive Evolutionary Spectrum Analysis for Narrow band Signals. ICASSP 87, Dallas, TEXAS.

[9] J. M. T. ROMANO. (1987). Localisation de frequences brvitees par filtrage adaptatif et implémentation d'algorithmes des MCR. Thése de doctorat.Univ. de Paris-sud, Orsay France.

[10] A. NEHORAI and B. PORAT (1986). Adaptive comb filtering for harmonic signal enhancement. IEEE Trans. ASSP, V 34, N 5, October 1986.

[11] I. D. LANDAU. A Feedback system approach to Adaptive Filtering. IEEETrans. IT, V 30, March 1984.

[12] R. M. JOHNSON et al. Exponential convergence of RLS with exponential forgetting favtor. System and control letters, V2, N2, August 1982.

Volume $\mathrm{V}$ begins on page 2493 